\title{
11 Index des noms de personnes et de lieux cités dans le texte d'Anjorrant Bourré
}

La forme française moderne (si elle existe) tient lieu d'entrée et elle est en italiques ; les formes du texte sont en caractères romains après les deux points. Pour éviter toute confusion ou pour identifier un personnage peu connu, une précision a parfois semblé nécessaire : une glose a alors été ajoutée entre crochets, avec référence à l'article de la Real-Encyclopädie $(R E),{ }^{1}$ de sa refonte $(N R E)^{2}$ ou, le cas échéant, de l'ouvrage The Magistrates of the Roman Republic $(M R R) ;^{3}$ nos identifications suivent majoritairement celles des éditions Testard, Winterbottom et Griffin \& Atkins.

Les entrées en gras correspondent à des noms absents du texte de Cicéron, mais ajoutés par Anjourrant Bourré ; quand le personnage est cité par ailleurs par Cicéron dans le texte, seule la référence de l'entrée d'Anjourrant est en gras.

Les entrées précédées du symbole † correspondent aux cas où la personne identifiée par Bourré semble diverger de celle à laquelle Cicéron paraît se référer, en raison d'une confusion ou d'une erreur de lecture (de sa part ou de la part du transcripteur de l'archétype).

Le * renvoie à une autre entrée dans l'index.

Enfin, en raison de leur fréquence, nous n'avons pas relevé les attestations de Rome et Romains.

Accius (Lucius) [écrivain, auteur de tragédies, $I I^{e}$ s.- $I^{\text {er }}$ s. ; RE, 1 / NRE, 1] : Actius (III.84.4-5, III.102.12, III.106.3).

Acilius (Caius) [sénateur et historien, $I I^{e}$ s. ; RE, 4 / NRE, 2] : Actilius, qui escript en grec l'ystoire des Romains (III.115.1).

Actreus [personnage non identifié, sans doute confusion avec le titre d'une des tragédies d'Accius, l'«Atrée»] : Actreus (III.102.12*).

${ }^{\dagger}$ Actuereus : cf. «Atrée».

Aelius (Quintus Aelius Tubero) [consul en 11 ; RE, 155 / NRE, I 16 / MRR, 2, 526] :

Quinctus Tyberio (II.77.5), Quintus Tuberon (III.63.2).

Aemilius (Mamercus Aemilius Lepidus Liuianus) [consul en 70 ; RE, 80 / NRE, I.13 / MRR, 3, 8-9] : Mamercus (II.58.2).

Aemilius (Lucius Aemilius Paullus) [consul en 219 ; RE, 118 / NRE, I.31 / MRR, 2, 528] : Paulus (III.114.1).

1 Pauly/Wissowa 1894-1972.

2 Cancik/Schneider 1996-2003.

3 Broughton 1968.

Ә Open Access. (C) 2019 Olivier Delsaux, published by De Gruyter. (c) BY-NC-ND This work is licensed under the Creative Commons Attribution-NonCommercial-NoDerivatives 4.0 License. 
Aemilius (Lucius Aemilius Paullus Macedonicus) [consul en 182 et en 168 ; RE, 114 / NRE, I.32 / MRR, 2, 528] : Paulus (I.116.3, II.76.7).

Aemilius (Marcus Aemilius Scaurus) [consul en 115 ; père du précédent ; RE, 140 / NRE, I.37 / MRR, 2, 528] : Marcus Staurus (I.76.26), Marcus Scaurus (I.108.1). Aemilius (Marcus Aemilius Scaurus) [fils du précédent ; RE, 141 / NRE, I.38 / MRR, 2, 528] : Staurus (I.138.6, II.57.8).

Agamemnon [fils d'Atrée et roi de Mycènes] : Agamenon (III.95.1-3).

Agésilas [roi de Sparte, $V^{e}-I V^{e}$ s. ; RE, 4 / NRE, 4] : Angesilaus (II.16.2).

Agis IV [roi de Sparte, III ${ }^{e}$ s. ; RE, 4 / NRE, 4] : Hagim (II.80.3).

Ajax [fils de Télamon] : Ayax (I.113.3), Ajax (III.97.1, III.98.1).

Albucius (Titus Albucius) [sénateur romain, $I I^{e}$ s. ; RE, 2 / NRE, 2 / MRR, 2, 529] :

Marcus Albucius (II.50.1-2).

Alexandre de Phères [tyran de Thessalonie, fils de Jason de Phères, IV ${ }^{e}$ s. ; RE/

NRE, 5] : Alixandre Phereus (II.25.6, II.26.2).

Alexandre le Grand : Alixandre (I.21.4, I.157.8-18, II.48.6, II.51.3, II.53.3),

Alexandre (II.16.2).

Alexandrie : Alixandrie (II.82.2, III.51.7, III.67.2), Alixandre (III.50.3).

${ }^{\dagger}$ Amenes le Galle : Amenes le Galle (III.112.18 [ad Anienem Galli]).

Annicériens : Amicenes (III.116.4).

Antigone I Monophtalmos [général de Philippe II de Macédoine et d'Alexandre le Grand, IV S. ; RE/NRE, 3] : Antigon (II.48.6).

Antipater [général de Philippe II de Macédoine et d'Alexandre le Grand, IV ${ }^{e}$ s. ; $R E, 12 / N R E, 2]$ : Antipater (II.48.6).

Antipater de Tarse [philosophe stö̈cien, $I I^{e}$ s. ; RE, 26/NRE, 10] : Antipater (III.51.1-2, III.52.1, III.53.1, III.54.6, III.56.1, III.91.4, III.92.2).

Antipater de Tyr [philosophe stö̈cien, ${ }^{\text {er }}$ s. ; RE, 27/NRE, 11] : Antipater Tirius (II.86.1).

Antonius (Marcus Antonius) [consul en 97 ; RE, 28 / NRE, I.7 / MRR, 2, 531] : Marcus Anthonius (II.49.4, III.67.10-13).

Apollon Pythius : Appolo (I.76.18), Appolo Pithius (II.77.14).

Aquilius (Manius Aquilius) [consul en 101 ; RE, 11 / NRE, I.4 / MRR, 2, 532] : Manilius (II.50.2).

Aquilius (Caius Aquilius Gallus) [juriste ; RE, 23 / NRE, I.12 / MRR, 2, 533] : Acquilius (III.60.3, III.61.1-3).

Aratus de Sicyone [homme politique grec, III ${ }^{e}$ s. ; RE/NRE, 2] : Aratus Sitionius (II.81-83).

Arceus : cf «Atrée».

Aréopage : cf. «Ariopagitans ».

Ariopagiteins : Ariopagitans (I.75.2 [Areopagitas], I.75.6 [Areopagum]).

Aristide [général et homme politique athénien, $\left.V I^{e}-V^{e} s . ; R E / N R E, 1\right]$ : Aristodes (III.16.3, III.49.2-4), Aristides (III.87.1). 
Aristippe de Cyrène [compagnon de Socrate, $V^{e}-I V^{e}$ s. ; RE, 8 /NRE, 3] : Aristipus (I.148.2, III.116.4).

Aristodes : cf. «Aristide».

${ }^{\dagger}$ Ariston de Chio [philosophe stoïcien, III ${ }^{e}$ s. ; RE, 56 / NRE, 7] : Aristote (I.6.3).

Aristote : le philozophe (0.11), Aristote (I.4.3, II.57.2, III.35.6) ; cf. «Ariston de Chio ».

Arpiniens : Arpinians (I.21.13).

Assiriens : Assiriens (I.26.5).

Athènes : Athenes (0.19, I.1.1, II.64.13), Atheines (II.71.10, II.86.1, III.6.4-6,

III.16.5, III.48.1, III.48.2, III.49.2, III.54.7, III.87.1, III.121.5), Athaye (III.46.4

[L Grece, $\mathrm{V}_{1}$ Athenie, P Athenes]).

Athéniens : Atheniens (I.75.1-4, I.84.6-8, I.86.1, III.46.6-9, III.48-49).

Atilius (Marcus Atilius Regulus) [consul en 267 et en 256 ; RE, 51 / NRE, II.21 /

$M R R$, 2, 534] : Regulus (I.38.8, I.39.3, I.39.7, III.100, III.101.1, III.102.12,

III.103-105, III.110, III.113.1, III.115.6), Marcus Actilius Regulus (III.99).

Atilius (Sextus Atilius Serranus) [consul en 136 ; RE, 69 / NRE, I.29 / MRR, 2,

536] : Sextus Actilius (III.109.14).

${ }^{\dagger}$ Arceus : «Atrée».

Arpiniens : Arpinians (I.21.13).

${ }^{\dagger}$ Atrée [roi de Mycènes, père d'Agamemnon] : Actuereus (I.97.4), Arceus (III.106.3).

Aufidius (Canius Aufidius Orestes Aurelianus) [préteur en 77 ; RE, 32 / NRE, I.8 / $M R R, 2,535]$ : Horresteus (II.58.7).

${ }^{\dagger}$ Auguste (Caius Octavius Imperator Caesar Augustus) : Cesar (I.112.2).

Augustin (saint) : saint Augustin (I.157.8).

${ }^{\dagger}$ Bardylis [roi des Illyriens, $I V^{e}$ s.] : Bargulus Illirius (II.40.6).

Bias de Priène [philosophe et homme politique grec, l'un des "septs sages" de Grèce, $V I^{e}$ s. ; RE/NRE, 2] : Bians (II.5.4, III.16.5).

Brutus : cf. «Iunius ».

Caecilius (Quintus Caecilius Metellus Macedonicus) [consul en 143 ; RE, 94 / NRE,

I.27 / MRR, 2, 539] : Quintus Metellus (I.87.3).

Caecilius (Quintus Caecilius Metellus Numidicus) [consul en 109 ; RE, 97 / NRE,

I.30 / MRR, 2, 539] : Quintus Metellus (III.79.5-9, III.81.6).

Caelus (montagne de Rome) : Celle (III.66.1).

Callicratidas [général spartiate, $V^{e}$ s. ; RE, 1] : Callicratidas (I.84.6, I.109.4).

Calliphon [philosophe hellénistique, III ${ }^{e}$ s. ; RE, 3] : Calipho (III.119.2).

Calpurnius (Publius Calpurnius Lanarius) [romain, ${ }^{\text {er }}$ s. ; RE, 49/NRE, I.7] : Publius Calphurnius (III.66.3-4).

Calpurnius (Lucius Calpurnius Piso Frugi) [consul en 133 ; RE, 96 / NRE, III.1 / $M R R, 2,542]$ : Lucius Pizo (II.75.8). 
Calypso [nymphe] : Calipsis (I.113.1).

Cannes : Cannez (III.47.8, III.113.1).

Canius (Gaius Canius) [personnage inconnu par ailleurs, ${ }^{\text {er }}$ s. ; RE Suppl. I.274] : Cannius (III.58).

Carthage : Cartage (I.35.5, I.76.28, II.76.8), Cartaige (III.99.8, III.100.14, III.110.3, III.111.3).

Carthaginois : Affricans (I.38.7-8, I.39.5, I.39.7, I.108.5, III.99.5, III.108.4, III.110.6), Affriquans (I.38.11), Cartaginiens (III.3.4), Carthaginiens (III.102.14).

Cassandre [fils d'Antipater, IV ${ }^{e}-I I I^{e}$ s. ; RE, 2] : Cassandre (II.48.6).

Catillus : cf. «Lutatius ».

Celtibères : Celtiberes (I.38.5).

Cesar : cf. «Iulius ».

Chilon de Sparte [philosophe, un des «sept sages » de Grèce, VI ${ }^{e}$ s. ; RE/NRE, 1] : Chilon (III.16.5).

Chrysippe de Soloi [philosophe stoïcien, III ${ }^{e}$ s. ; RE/NRE, 2] : Crisipus (III.42.3).

Cimbres : Cimbriens (I.38.5).

Cimon [homme d'État athénien, $V I^{e}$ s.] : Symon (II.64.13).

Circé [fille du Soleil] : Circes (I.113.1).

Claudius (Titus Claudius Centumalus) [personnage inconnu par ailleurs ; RE, 107] : Titus Claudius Centumanus (III.66.1), Titus Claudius (III.66.5-8).

Claudius (Marcus Claudius Marcellus) [consul entre 222 et 208 ; RE, 220 / NRE, I.11 / MRR, 2, 546] : Marcus Marcellus (I.61.6).

Claudius (C. Claudius Ap. f. Pulcher) [consul en 92 ; RE, 302 / NRE, I.23 / MRR, 2, 547] : Quinctus Claudius (II.57.5).

Cléobule de Lindos [philosophe et homme politique, VII $-V I^{e}$ s., l'un des «sept sages » de Grèce] : Cleobotus (III.16.5).

Cléombrote [roi de Sparte, IV ${ }^{e}$ s. ; RE, 2] : Cleombratus (I.84.9).

Collatinus : cf. «Tarquinius».

Conon [homme d'État athénien, $V^{e}-I V^{e}$ s. ; RE, 3] : Conon (I.116.6).

Corinthe : Corinthe (III.46.4, III.46.4).

Corinthiens : Corinthiens (I.35.6).

Cornelius (Publius Cornelius Lentulus Spinther) [consul en 57 ; RE 238 / MR, I.54 / MRR, 2, 554] : Publius Lentulus (II.57.8).

Cornelius (Publius Cornelius Scipio Aemilianus Africanus Minor) [consul en 147 et en 134 ; RE 336 / NRE, I.71 / MRR, 2, 555] : Scipio l'Affrican (I.76.28, I.116.3, II.76.1), Publius l'Affrican (I.87.3, I.90.9, I.108.2).

Cornelius (Publius Cornelius Scipio Africanus Maior) [consul en 205 et en 194 ; RE 335 / NRE, I.70 / MRR, 2, 555] : Scipio l'Affrican (II.80), Scipio, qui fut le premier appelé l’Affricant (III.1.1), l’Affrican (III.1.6, III.2.1-4, III.4.1), Scipio (III.3). 
Cornelius (Cneius Cornelius Scipio Caluus) [consul en 222 ; RE 345 / NRE, I.77 / MRR, 2, 556] : Publius Scipio (I.61.6 [Cn. et P. Scipiones ; télescopage de Cneius Cornelius Scipio Caluus et de Publius Cornelius Scipio Africanus Maior]).

Cornelius (Publius Cornelius Scipio Nasica Serapio) [consul en 138 ; père du suivant ; RE 354 / NRE, I.84 / MRR, 2, 556] : Publius Nasica (I.76.28)

Cornelius (Publius Cornelius Scipio Nasica Serapio) [consul en 111 ; fils du précédent ; RE 355 / NRE, I.85 / MRR, 2, 556] : Publius Scipio Nasica (I.109.8).

Cornelius (Lucius Cornelius Sulla Felix) [consul en 88 ; RE 492 / NRE, I.90 / MRR, 2, 557] : Lucius Scilla (I.43.4, I.109.3, II.27.2-3, II.83.4, III.87.7).

Cornelius (Publius Cornelius Sulla) [consul en 65 ; RE 386 / NRE, I.89 / MRR, 2, 557] : Lucius Scilla (II.29.2).

Crassus : cf. «Licinius».

Cratidianus : cf. «Marius».

Cratippe de Pergame [philosophe péripatéticien, ${ }^{e r}$ s. ; RE, 3] : Cratipus (0.19, I.1-2, III.5.3-4, III.33.5, III.121.3).

Crête : Crete (I.76.20).

Curio : cf. «Scribonius ».

Curius (Marcus Curius Dentatus) [consul en 290, 275 et 274 ; RE 9 / NRE, 4 / $M R R, 2,558]$ : Marcus Curio (II.71.13).

Cyrénaïques : Cirenayques (III.116.4).

Cyrsilus [Athénien lapidé pour avoir inviter à céder à Xerxès, $V^{e}$ s.] : Circilus (III.48.2-3).

Cyrus [général et homme d'État, fondateur de l'empire perse, $V I^{e} s$.] : Sirus (II.16).

Damoclès [ami de Denis de Syracuse ; RE, 6] : Democles (I.23.6-20).

Damon [pythagoricien de Syracuse, $I^{\text {er }}$ ou II ; RE, 18] : Damon (III.45.1).

Darius (Darius $I^{\text {er }}$ [roi de l'empire perse, $\mathrm{VI}^{e}-\mathrm{VII}^{e} \mathrm{~s}$.] ?) : Daire (I.148.6).

David [roi d'Israël] : David (I.21.8-11, I.26.7).

Decius (Publius Decius Mus) [le père [consul en 340 ; RE 16 / NRE, I.2 / MRR, 2, 559] et le fils [consul en 295 RE 17 / NRE, I.3 / MRR, 2, 559] : Decius Cesar (I.61.6), Decius l'oncle et Decius son nepveu (III.16.1).

Démétrius de Phalère [philosophe péripatécien, IV ${ }^{e}$ s. ; RE, 85 / NRE, I.4] : Demetrius Phalerius (I.3.3, II.60.6).

Démétrius I Poliorcète [homme d'État athénien, IV $-I I I^{e}$ S. ; RE, 33 / NRE, I.2] : Demetrius (II.5.7, II.26.4).

Démosthène [orateur grec, IVe s. ; RE/NRE, 2] : Demostenes (I.4.2, II.47.4).

Denis $I^{e r}$ de Syracuse [tyran de Syracuse, $V^{e}-I V^{e}$ s. ; RE, 1] : Denis le tirant

(II.23.6-21, II.25.1, III.45.2, III.45.7).

Diane [déesse] : Dyana (III.95.1-2).

Dicéarque de Messine [philosophe péripatéticien, $I V^{e}-I I I^{e}$ s. ; RE, 3] : Dyarche (II.16.6). 
${ }^{\dagger}$ Dinomaque [philosophe hellénistique, III ${ }^{e}$ s. ?] : Dynomarchus (III.119.2).

Diogènes de Babylone [philosophe stö̈cien, III ${ }^{e}-I^{e}$ s. ; RE, 45 / NRE, 15] : Dyogenes le Babilonien (III.51.1-4), Dyogenes (III.52.6, III.53.3-9, III.91.2-4, III.92.2), Diogenes (III.55.1, III.56.1).

Dion de Syracuse [beau-frère de Denis $I^{e r}$ de Syracuse, $V^{e}-I V^{e}$ s. ; RE, 2 / NRE, I.1] : Seracusius et Dyogenes (I.155.6).

Dionides [nom du pirate qui interpelle Alexandre le Grand sur sa richesse] : Dyonides (I.157.8-19).

Drusus : cf. «Liuius».

${ }^{\dagger}$ Dyogenes : Dyogenes (I.155.6) ; cf. «Dion de Syracuse».

Éaque [grand-père d'Achille] : Eacius (I.97.3).

Égine (île grecque) : Egine (III.46.6).

Éginiens : Eginiens (III.46.9).

Égypte : Egipte (III.49.2, III.63.1).

Emilius : cf. «Maelius ».

Ennius (Quintus Ennius) [poète romain, $I I I^{e}-I I^{e}$ s.] : Ennius (I.26.2, I.52.5, I.84.11, II.23.2, II.62.4, III.62.8-9, III.104.8).

Epaminondas [général et homme d'État de Thèbes, $V^{e}-I V^{e} s$. ; RE/NRE, 1] : Epannimunde (I.84.9) ; Thebane et Epaminunde (I.155.5).

Epirotes : Epirotes (II.38.6).

Eques : Eques (I.35.3).

Étéocle [fils d'Edipe et de Jocaste] : Etheocles (III.82.13).

Euripide [tragédien athénien, $V^{e} s$.] : Euripedes (III.82.13), Euripedes (III.108.3).

Fabius (Quintus Fabius Labeo) [consul en 183 ; RE 91 / NRE, I.20 / MRR, 2, 562] :

Fabius Labeo (I.33.13).

Fabius (Quintus Fabius Maximus Verrucosus) [consul en 233, 228, 215, 214, 209 ; RE 116 / NRE, I.30 / MRR, 2, 563] : Quintus Maximus (I.84.10-12, I.108.6).

Fabricius (Caius Fabricius Luscinus) [consul en 282 et en 278 ; RE 9 / NRE, 3 / $M R R, 2,564]$ : Fabrius (III.16.3), Fabricius (III.86.3, III.87.1).

Fimbrias : cf. "Flauuius ».

Flauius (Caius Flauius Fimbria) [consul en 104 ; RE 87 / NRE, I.4 / MRR, 2, 566] : Fimbrias (III.77.1).

Flauius (Publius Flauius Vegetius Renatus) [historien romain, $\left.I V^{e}-V^{e} s.\right]$ : Vegece (II.38.6).

${ }^{\dagger}$ Fufius (Lucius Fufius) [orateur romain, $I^{\text {er }}$ S. ; RE, 5 / NRE, I.2 / MRR, 2, 566] : Lucius Fusius (II.50.2).

${ }^{\dagger}$ Furius (Lucius Furius Philus) [consul en 136 ; RE 78 / NRE, I.28 / MRR, 2, 572] : Lucius Fusius (III.109.14).

Fusius : cf. «Furius » et « Fufius ».

Jean de Galles [franciscain anglais, philosophe et théologien, auteur du «Breuiloquium », XIII ${ }^{e}$ s.] : Gallensis (I.157.5), Galence (II.23.6). 
Gayus Cesar : cf. «Iulius».

Goliath [géant philistin] : le geyant Golias (I.21.6-11).

Gracus : cf. «Sempronius ».

Gygés [roi de Lydie, VII ${ }^{e}$ s. ; RE, 2 / NRE, 1] : Giges (III.38.1-4, III.78.1).

Hannibal [général carthaginois, III ${ }^{e}$ s.] : Hannibal (I.108.5), Hanibal (III.113-114).

Hécaton de Rhodes [philosophe stö̈cien, $I^{e r}$ s. ; RE/NRE, 1] : Heccaton le Rodian

(III.63.1-6, III.89.1, III.89.7-9, III.90.5, III.90.7-10).

Helinand de Froidmont [écrivain et historien français, XII $\left.{ }^{e}-X I I I^{e} s.\right]$ : Helinandus (II.34.3).

Hercule [fils de Jupiter] : Hercules (I.118.1, I.118.6).

Herniques : Herniques (I.35.3).

Hérodote d'Halicarnasse [historien grec, $V^{e}$ s.] : Erodotus (II.41.1).

Hippolyte [fils de Thésée et d'Hyppolité] : Ypolite (I.32.2-5), Ypolite (III.94).

Homère : Homerus (III.97.2).

Horresteus : cf. «Aufidius ».

Hortensius (Quintus Hortensius Hortalus) [consul en 69 ; RE 13 / NRE, 7 / MRR,

2, 573] : Hortensius (III.73.5-6, III.74.2).

Hostilius (Caius Hostilius Mancinus) [consul en 137 ; RE 18 / NRE, I.8 / MRR, 2,

573] : Gayus Mantinus (III.109.11, III.109.16).

Isidore de Séville [évêque et écrivain espagnol, $V^{e}-V I^{e}$ s.] : Ysodore (I.151.11).

Isocrate [orateur athénien, $V^{e}-I V^{e} s . ; R E, 2$ / NRE, 1] : Socrates (I.4.3).

Israël : Israel (I.26.7).

Iulius (Caius Iulius Caesar) [empereur romain, ${ }^{\text {er }}$ s. ; RE, 131 / MRR, 2, 574] :

Gayus Cesar (I.26.4, I.43.4, I.61.6, II.29.1, II.84.10, III.3.1, III.82.6, III.83.2,

III.83.5), Cesar (I.112.6-7, II.2.4, II.27.7, II.28.4, II.83.4), Julius Cesar (II.45.6,

II.23.4, II.26.2).

${ }^{\dagger}$ Iulius (Caius Iulius Caesar Strabo Vopiscus) [orateur romain, I ${ }^{\text {er }}$ S. ; RE, 135 /

NRE, I.11 / MRR, 2, 574] : Gayus Cesar (I.108.1), Cesar (I.133.10).

Iunius (Lucius Iunius Brutus) [premier consul de Rome, VI ${ }^{e}$ s. ; RE, 46a / NRE,

I.4 / RE Suppl. V, 356 / MRR, 2, 576] : Brutus (III.40.4).

Iunius (Marcus Iunius Brutus) [juriste romain, ${ }^{\text {er }}$ S. ; RE, 50 / NRE, I.10 / MRR, 2,

576] : Marcus Brutus (II.50.8).

Iunius (Marcus Iunius Pennus) [tribun de la plèbe en 126 ; RE, 123 / I.24 / MRR,

2, 577] : Pennius (III.47.1).

Iunius (Decimus Iunius Silanus) [consul en 62 ; RE, 163 / NRE, I.30 / MRR, 2,

577] : Sillanus (II.57.7).

Jason de Phères [tyran de Thessalie, $I V^{e}$ s. ; RE/NRE, 3] : Phereus et Jazon

(I.108.8), Jason (III.47.5).

Jazon : cf. «Jason de Phères ».

Jérôme [saint, $\left.I V^{e}-V^{e} s.\right]$ : Jerosme (II.71.10). 
Jugurtha [roi des Numides, II ${ }^{e}$ s.] : Jugurtha (I.121.8-14, III.79.5).

Jupiter : Jupiter (I.118.6, III.102.2-4, I.104.8-10, I.105.1).

Lacédémoniens : Lacedemoniens (I.64.2, I.76.2, I.76.8-15, I.84.6, II.26.5, II.77.15, II.80.1, II.80.7, III.49.2).

Laciades : Laciades (III.64.13-14).

Laelius (Caius Laelius Sapiens) [consul en 140 ; RE, 3 / NRE, I.2 / MRR, 2, 578] : Gayus Lelius (I.90.3, I.108.2, II.40.9, III.16.5).

Laomédon [roi de Troie, père de Priam] : Laomedon (III.47.5).

Latins : Latins (I.38.7, III.112.21).

Lentulus : cf. «Cornelius (Publius Cornelius Lentulus Spinther)».

Licinius (Lucius Licinius Crassus) [consul en 95 ; RE, 55 / NRE, I.10 / MRR, 2, 579] : Lucius Crassus (I.108.1, I.133.8, II.47.2, II.49.4, II.57.6, II.63.8, III.47.3, III.67), Publius Crassus le riche (II.57.4).

Licinius (Marcus Licinius Crassus Diues) [consul en 70 ; RE, 68 / NRE, I.18 / MRR, 2, 580] : Mussius Crassus (I.25.3), Marcus Crassus (I.109.3, III.73.6, III.74.2, III.75.6, III.78.2).

Licinius (Lucius Licinius Lucullus) [consul en 74, frère aîné du suivant ; RE, 104 / NRE, I.26 / MRR, 2, 580] : Lucius Luculus (I.140.4), les deux Luculles (II.50.1), Luculus, le pere et aussi le filz (II.57.7).

Licinius (Marcus Licinius Lucullus) [consul en 73, frère du précédent ; RE 109 / I.27 / MRR, 2, 580] : les deux Luculles (II.50.1), Luculus, le pere et aussi le filz (II.57.7).

Lucius : cf. «Marcius».

Lutatius (Quintus Lutatius Catulus) [consul en 102, père du précédent ; RE, 7 / NRE, I.3 / MRR, 2, 583] : Quintus Catillus (I.76.26, I.109.6), Catullus le pere (I.133.6).

Lutatius (Quintus Lutatius Catulus) [consul en 78, fils du précédent ; RE, 8 / NRE,

I.4 / MRR, 2, 583] : Quintus Catillus (I.109.6), Catullus le fils (I.133.6).

Lutatius (Marcus Lutatius Pinthia) [chevalier romain, ${ }^{\text {er }}$ s. ; RE 21 / MRR, 2, 580] : Marchus Lutharius (III.77.1-4).

Lycurgue [législateur de Sparte, VIII ${ }^{e}$ s. ; RE, 7 / NRE, 4] : Ligurgus (I.76.1-2, I.76.15).

Lydie [région d'Asie mineure] : Lidie (III.38, III.78.1).

Lysandre [général de Sparte, $V^{e}-I V^{e} s . ; R E / N R E$, 1] : Lisander (I.76.1, I.109.4). Lysandre [homme politique qui soutint les réformes du roi de Sparte Agis IV, III ${ }^{e}$ s. ; RE/NRE, 2] : Lisander Ephorus (II.80.2).

Lysis de Tarente [philosophe pythagoricien, $I V^{e}$ s. ; RE, 2 / NRE, 1] : Lisias Pithagoreus (I.155.5).

Maccius (Titus Maccius Plautus) [auteur de comédies, III ${ }^{e}-I^{e}$ s.] : Plautus (I.104.3). 
Macedoine : Macedoyne (I.37.1) ; cf. «Philippe de Macédoine.

Macedoniens : Macedoniens (II.26.4, II.53.3).

Macrobe (Ambrosius Theodosius Macrobius) [écrivain latin, $I V^{e}-V^{e} s$.] : Macrobe (II.23.6).

Maelius (Quintus Maelius) [conjecture éditoriale ; mss "Aemilius »] [tribun de la plèbe en 321 ; RE/NRE, 1] : Quintus Emilius (III.109.10).

Mantinus : cf. «Hostilius ».

Mamercus : cf. « Aemilius».

Manilius : cf. «Aquilius».

Manlius (Lucius Manlius Capitolinus Imperiosus) [dictateur en 363, père du suivant ; RE, 54 / NRE, I.11 / MRR, 2, 586] : Lucius Manlius (III.112).

Manlius (Titus Manlius Imperiosus Torquatus) [consul en 347, 344 et 340, fils du précédent ; RE, 57 / NRE, I.12 / MRR, 2, 586] : Titus (III.112).

Marcellus : cf. «Claudius».

Marcius (Lucius Marcius Philippus) [consul en 91 ; RE, 75 / NRE, 91 / MRR, 2, 588] : Lucius Philippus (I.108.1), Lutius Philipus (II.59.2), Philippus (II.73.26), Philipus Lucius (III.87.4).

Marius (Caius Marius) [consul en 107, 104, 103, 102, 101, 100, 86 ; RE, 14 / NRE, I.1 / MRR, 2, 589] : Gayus Marus (I.76.25, II.27.3-4, III.79-81).

Marius (Marcus Marius Gratidianus) [préteur en 85 ; RE, 42 / NRE, I.7 / MRR, 2, 589] : Marcus Marius Cratidianus (III.67.4).

Marseilles : Massillie (II.28.2).

Maximus : cf. «Fabius ».

Mèdes : ou pays de Medie (II.41.1).

Mégare : Megare (II.5.7).

Metellus : cf. «Caecilius ».

${ }^{\dagger}$ Métrodore de Lampsaque [philosophe, disciple d'Épicure, IV ${ }^{e}-I I{ }^{e}$ s. ; RE 16 / NRE, 3] : Metheodorus (III.117.3).

Micipsa [roi des Numides, $I I^{e}$ s.] : Mycipsa (I.121.7-14).

Minos [fils de Zeus et d'Europe] : Minos (I.97.3).

Minucius (Lucius Minucius Basilus) [homme politique romain, partisan de Sylla, $I^{e r}$ s. ; RE, 38 / NRE, I.4 / MRR, 2, 592] : Lucius Municius Basilius (III.73.4), Lucius Municius (III.73.8, III.74.1-2).

Mucius (Publius Mucius Scaeuola) [consul en 133 ; RE, 17 / NRE, I.5 / MRR, 2, 593] : Publius Mucius (II.47.1).

Mucius (Quintus Mucius Mancia) [? ; le passage pourrait faire référence à Quintus Mucius Scaeuola Augur] : Quinctus Mucius (I.109.7).

Mucius (Quintus Mucius Scaeuola Pontifex) [consul en 95 ; RE, 22 / NRE, I.9 / $M R R, 2,593]$ : Quinctus Mucius (I.116.1, II.57.6, III.47.3), Quintus Scevola (III.62-63, III.70.4). 
${ }^{\dagger}$ Mummius (Lucius Mummius Achaicus) [consul en 146 ; RE, 7a / NRE, I.3 / MRR, 2, 593] : Lucius Munilius (II.76.9).

Munilius : cf. «Mummius».

Mussius : cf. «Licinius».

Nabuchodonosor [empereur de Babylone, $\mathrm{VII}^{e}-\mathrm{VI}^{e}$ s.] : Nabugodenosor (I.26.5).

Napolitains : Neapolins (I.33.9), Neapolitains (I.33.10).

Nasica : cf. «Cornelius ».

Neptune [divinité romaine] : Neptunus (I.32.2-5, III.94.10-12).

${ }^{\dagger}$ Nicoclès [tyran de Sicyone, III ${ }^{e}$ S. ; RE, 5 / RE Suppl. VII, 570 / NRE, 4] : Niocodes (II.81.1-4).

Noé [patriarche] : Noé (II.16.7).

Nolains : Nolains (I.33.9).

Norbanus (Gaius Norbanus) [consul en 83 ; RE, 5 / NRE, I.1 / MRR, II, 595] : Gay le Norbain (II.49.5).

Numance : Numense (I.35.5).

Numantins : Numenciens (III.109.12-13).

${ }^{\dagger}$ Numicius (Tiberius Numicius) [tribun de la plèbe en 320 ; RE, 3 / NRE, 1] : Tiberius Municius (III.109).

Numides : Numydes (I.121.7).

Octauius (Cneius Octauius) [consul en 165 ; RE, 17 / NRE, I.4 / MRR, II, 595] : Gueyus Octavius (I.138.3).

Octauius (Marcus Octauius) [tribun de la plèbe entre 123 et 78 ; RE, 37 / NRE, I.10] : Marcus Octavius (II.72.6).

Ortensius : cf. «Hortensius».

Panétius de Rhodes [philosophe stoïcien, II ${ }^{e}$ s.] : Panecius (I.7.1, I.9.1, I.10.1, I.10.5, I.90.9, I.152.3, I.60.8, II.16.1, II.35.7, II.51.7, II.60.5, II.86.1-2, II.88.2, III.7.1, III.8-11, III.12.1, III.18.3, III.33.1, III.34.1, III.34.8-9, III.63.1).

Papius (Caius Papius) [tribun de la plèbe en 65 ; RE/NRE, 5 / MRR, 2, 599] :

Papius (III.47.1).

Paulus : cf. «Aemilius».

Pausanias [général de Sparte, $V^{e}$ s. ; RE, 5 / NRE, 1] : Pausania (I.76.1).

Pélops [fils de Tantale et père d'Atrée] : Pelope (III.84.6).

Pénélope [femme d'Ulysse] : $\alpha$ Penelopes (III.97.5 [R $\left.{ }^{\circ}\right]$ ).

Pennius : cf. «Iunius».

Périandre de Corinthe [tyran de Corinthe, l'un des "sept sages " de Grèce, VII ${ }^{e}$

s.] : Periandus (III.16.5).

Periclès [homme d'État athénien, $V^{e}$ s.] : Periclis (I.108.4, I.144.3), Perules (II.16.2).

Perses : Perses (I.37.1, III.48.1-2).

Phaéton [fils du Soleil] : Pheton (III.94.1-5). 
Phalaris [tyran d'Agrigente, $\mathrm{VI}^{e}$ s.] : Phalaris (II.26.1, III.29.4, III.30.2, III.32.1). Phereus : cf. «Jason de Phères ».

Philippe II de Macédoine [roi de Macédoine, père d'Alexandre le Grand, IV ${ }^{e}$ s. ;

$R E, 7$ / $R E, 4]$ : Phelippe le roy de Macedoine (I.90.4), Phelippe (II.48.6, II.53.4), Philipe (II.48.6).

Philipus : cf. «Marcius».

Phintias [ami du pythagorien de Syracuse, Damon, $I^{e r}-I I^{e}$ s. ; RE, 3] : Pinicias (III.4.1).

'Phiro : cf. «Pyrrhon d'Élis ».

${ }^{\dagger}$ Phirus : cf. «Pyrrhus».

Pittacos de Mytilène [homme politique grec, un des "sept sages" de Grèce, $V I^{e}$ siècle ; RE, 2] : Pitacus (III.16.5).

Platon [philosophe, $V^{e}-I V^{e}$ s.] : Platon (I.4.1, I.15.2, II.22.1, I.28.3, I.64.2, I.85.1, I.87.2, I.118.11, I.148.6, I.155.5, III.38.1, III.39.1).

Plautus : cf. «Maccius».

Polybe [historien grec, II ${ }^{e}$ s. ; RE, 1 / NRE, 2] : Polibius (III.113.4, III.114.8).

Pompeius (Cneius Pompeius Magnus) [général romain, $\mathrm{II}^{e}-\mathrm{I}^{\text {er }}$ S. ; RE, 15 / NRE, I.3 / MRR, 2, 603] : Gayus Pompeyus (I.76.25-26, I.78.2, II.57.9), Pompee (II.20.1, II.45.6, III.82.6).

Pompeius (Quintus Pompeius) [consul en 141 ; RE, 7 / NRE, I.1 / MRR, 2, 603] : Quintus Pompeius (III.109.17).

Pompeius (Cneius Pompeius Trogus) [historien romain, $I^{\text {er }}$ S. ; RE, 142 / NRE, III.3] : Tregus Pompeius (I.76.2).

Pompeius (Sextus Pompeius Virdoctus) [oncle de Cneius Pompeius Magnus, $\mathrm{II}^{e_{-}}$ $I^{\text {er }}$ S. ; RE, 18 / NRE, II.4 / MRR, 2, 610] : Sextus Pompeyus (I.19.3).

Pomponius (Marcus Pomponius) [tribun, IV ${ }^{e}$ s. ; RE, 8 / NRE, I.2 / MRR, 2, 694] : Marcus Pomponus (III.112.8).

Pontius (Caius Pontius) [chef de guerre samnite, $I I{ }^{e}-I I^{e}$ s. ; RE/NRE, 4] : Gayus Poncius (II.75.2).

Popilius (Marcus Popilius Laenas) [consul en 173 ; RE, 7 / NRE, I.4 / MRR, 2, 605] : Pompilius (I.36.4-6).

Porcius (Marcus Porcius Cato (maior) Censorius) [consul en 195 ; RE, 10 / NRE, I.6 / MRR, 2, 606] : Chaton (I.36.4, I.37.1, I.104.3, II.89.3-5, III.104.10), Marcus Catho (I.79.4, III.16.5), le grant Chaton (I.151.5), [le] vieulx Chaton (II.89.1).

Porcius (Marcus Porcius Cato Licinianus) [fils du précédent ; RE, 14 / NRE, I.9 / $M R R, 2,606]$ : le filz de Chaton (I.36.4, I.37.1).

Porcius (Marcus Porcius Cato (minor) Vticensis) [préteur en 54, arrière-petit-fils du Censorius ; RE, 12 / NRE, I.7 / MRR, 2, 606] : Marcus Catho (I.112.5, III.66.7-8), Chaton (III.88.2). 
Posidonius d'Apamée [philosophe stoïcien, $\left.I^{e}-I^{e r} ; R E / N R E, 3\right]$ : Possidonius (I.159.3), Possidoyne (III.8-10).

Postumius (Spurius Postumius Albinus) [consul en 334 et en 321 ; RE, ${ }^{*} 17$ / NRE, I.5 / MRR, 2, 607] : Spurius Postumus (III.109.1, III.109.8).

Priène [cité d'Asie mineure] : Prienne (II.5.4).

Ptolémée II Philadelphe [roi d'Égypte, IV III $^{e}$ s. ; RE, 19 / NRE, 3] : Ptholomee (II.82.3-7).

Publius l'Affrican : cf. «Cornelius ».

Pyrrhon d'Élis [philosophe sceptique, $I V^{e}-I I I^{e}$ s. ; RE/NRE, 1] : Phiro (I.6.3).

Pyrrhus [roi d'Épire, IV ${ }^{e}-I I I^{e}$ s.] : Phirus (I.38.7, II.18.7-9, II.26.4), Phirrus (III.86.1-4, III.87.1).

Pythagoras de Samos [philosophe et mathématicien, VI ${ }^{e}$ s.] : Pitagoras (I.56.4, I.108.4), Pithagoras (I.148.5, III.45.1).

Pythios [banquier à Syracuse, ${ }^{\text {er }}$ s. ; RE, 5] : Pithius (III.58-59).

Regulus : cf. «Atilius ».

Remus [frère de Romulus] : Remus (III.41.1-3).

Rhodes : Rodes (III.50.3-4, III.57.1, III.67.2).

Rhodien : Rodiens (III.52.11).

Romulus (frère de Remus) : Romulus (III.41.1-4).

Roscius (Sextus Roscius Amerinus) [fils d'un riche citoyen d'Amérie ; RE, 7 / NRE, I.2] : Sextus Roscius (II.51.11).

Rutilius (Publius Rutilius Rufus) [consul en 105 ; RE, 34 / NRE, I.3 / MRR, 2, 613] : Publius Rutilius (II.47.1-2, III.10.1).

Sabins et Samnites : Sadoniens (I.38.7 [Sabinis, Samnitibus]).

Salamine [île de l'Attique] : Sallanus (I.75.2).

†Sallanus : cf. «Salamine».

Sallustius (Caius Sallustius Crispus) [RE, 10 / NRE, II.3 / MRR, 2, 613] : Saluste (I.101.5, I.105.6).

Samnites : Sanites (II.71.13), Sannites (II.109.2-6).

Sardes : Sardiens (II.50.1).

${ }^{\dagger}$ Sarragoces : cf. «Syracuse».

Satrius (Marcus Satrius) [neveu adoptif de L. Minucius Basilus, I ${ }^{\text {er }}$ s. ; RE, 1 / NRE, I.1 / MRR, 2, 613] : Marcus Satrius (III.74.1-2).

Saul [roi d'Israël, XI ${ }^{e}$ s.] : Saul (I.21.6-11).

Scilla : cf. «Cornelius».

Scipio : cf. «Cornelius » et «Stilpon».

Scipio l'Affrican : cf. «Cornelius».

Scribonius (Caius Scribonius Curio) [consul en 76 ; RE, 10 / NRE, I.3 / MRR, 2, 614] : Gayus Curio (II.59.3, III.88.6).

Seius (Marcus Seius) [édile en 74 ; RE, 3 / MRR, 2, 614] : Marcus le viel (II.58.7). 
Sempronius (Caius Sempronius Gracchus) [tribun de la plèbe en 123 et 122 ; RE, 47 / NRE, I.11 / MRR, 2, 615] : Gayus Gracus (II.72.5).

Sempronius (Tiberius Sempronius Gracchus) [père du suivant, consul en 177 et 163 ; RE, 53 / NRE, I.15 / MRR, 2, 615] : Tiberius Gracus (II.43.5, II.80.8).

Sempronius (Tiberius Sempronius Gracchus) [fils du précédent, tribun de la plèbe en 133 ; RE, 54 / NRE, I.16 / MRR, 2, 615] : Titus Gracus (I.76.28, I.109.10).

Sénèque (Lucius Annaeus Seneca) : Seneque (II.5.7).

†Seracusius : cf. «Dion de Syracuse».

Sergius (Caius Sergius Orata) [homme d'affaires romain, $I^{\text {er }}$ S. ; RE, 33 / NRE, I.8] : Gayus Sergius (III.67.4-13).

Siciliens : Sticilliens (II.50.1).

Sicyone : Sitone (II.81-83).

Sillanus : cf. «Iunius».

Sitionius : cf. «Aratus».

Socrate : Socrates (I.90.3, I.104.3, I.108.3, I.134.2, I.148.2, II.43, II.87.2, III.11.7, III.77.4) ; cf. «Isocrate».

Socrate de Thèbes [?] : Socrates Thebanus (II.71.10).

Solon [RE/NRE, 1] : Solon (I.75.2-7, I.108.9, III.16.5).

Sophocle : Sophocles (I.144.3-8).

Sparte : Sparte (II.77.14).

Staurus : cf. «Aemilius».

${ }^{+}$Stilpon de Mégare [philosophe grec, $I I^{e} S$. ; RE/NRE, 1] : ung philozophe nomé Scipio (II.5.7).

Sulpicius (Caius Sulpicius Galus) [consul en 166 ; RE, 66 / NRE, I.14 / MRR, 2, 623] : Gayus Sulpicius (I.19.3).

Sulpicius (Publius Sulpicius Rufus) [tribun de la plèbe en 88 ; RE, 92 / NRE, I.19 / MRR, 2, 624] : Publius Sulpicius (II.49.5).

Syracuse : Sarragoces (III.58.2, III.59.2).

Tanguy (IV) du Chastel [grand maître d'hôtel du duc de Bretagne François II, puis chambellan du roi de France Charles VII] : Tanguy du Chastel, seigneur de Regnac (0.15).

Tantale [père de Pélops et fils de fils Jupiter] : Tantallus (III.84.6).

Tarquinius (Lucius Tarquinius Collatinus) [1 ${ }^{\text {er }}$ consul de Rome, VI ${ }^{e}$ s. ; RE, 8 / NRE, 9 / MRR, 2, 324] : Collatinus (III.40.4).

Tarquinius (Lucius Tarquinius Superbus) [dernier roi de Rome, VI ${ }^{e}$ s. ; RE, 11 / NRE, 12] : Traquinius (III.40.4), Tarquin (III.40.6).

Télémaque [fils d'Ulysse] : Thelematus (III.97.5).

Terentius (Publius Terentius Afer) [auteur de comédies, II ${ }^{e}$ s. ; RE, 36 / NRE, III.1] : Therence (I.30.2, I.137.7, I.150.7).

Terentius (Caius Terentius Varo) [consul en 216 ; RE, 83 / NRE, I.14 / MRR, 2, 625] : Varro (III.114.1). 
Thalès de Milet [philosophe et mathématicien grec, fait partie des "sept sages" de Grèce, $V_{I I}{ }^{e}-V I^{e}$ s.] : Tales (III.16.5).

Thébé [fille d'Alexandre de Phères, $I V^{e}$ s. ; RE, 4] : Thebes (II.25.6-8).

Thémistocle [général et homme d'État athénien, $\left.V I^{e}-V^{e} s.\right]$ : Themistodes (I.75.1-7, I.108.8, II.16.1, II.71.3, III.49).

Théophraste de Mytilène [philosophe, successeur d'Aristote, $I V^{e}-I I I^{e}$ s.] : Theofrastus (I.3.3, II.56.1, II.64.9, II.64.13).

Théopompe de Chio [philosophe et historien grec, IV s. ; RE, 9 / NRE, 3] : Theopompus (II.40.8).

Theostratus : cf. «Théophrase».

Thésée [roi mythique d'Athènes et père d'Hippolyte] : Theseus (I.32.2-5, III.94).

Thimochares d'Ambracie [serviteur de Pyrrhus, $I V^{e}-I I I^{e}$ s. ?] : Thimotare (II.18.8).

Timothée [homme politique et militaire athénien, IV ${ }^{e}$ s. ; RE, 3 / NRE, 5] : Thimotheus (I.116.6).

Trajan [empereur romain, $\left.I^{e r}-I I^{e} p C n\right]$ : Trajam (II.34.3-17).

Trézène [cité du Péloponèse] : Troezene (III.48.1).

Troie : Troie (I.38.6, III.47.5).

Troyens : Troians (I.38.6, III.95.1, III.97.1).

Tullius (Marcus Tullius Cicero) [le fils de l'auteur, $I^{\text {er }}$ s. ; RE, 30 / MRR, 2, 627] : Marcus Cicero (0.19), mon filz Marcus (0.11, I.15.1, I.78.1, II.1.1, III.121.1), mon filz Cicero (II.8.5, II.9.2, II.44.3-5, III.1.1, III.5.1, III.33.4).

Tusculains : Tusculains (I.35.3).

Ulysse : Ulixes (I.113.1, III.97.1, III.98-99).

Valerius Maximus [RE, 239 / NRE, III.5] : Valere (II.18.7, II.71.13, II.77.3).

Varro : cf. «Terentius ».

Vénus : Venus (III.10.1).

Veturius (Titus Veturius Caluinus) [consul en 334 et en 321 ; RE, ${ }^{*} 11$ / NRE, I.1 / $M R R, 2,633]$ : Titus Vecturius (III.109.1).

${ }^{\dagger}$ Viriathe [chef de guerre lusitanien, $I I^{e}$ s.] : Viriat le Liriatain (II.40.8), Viriat le Lisitam (II.40.9).

Vosques : Vulques (I.35.3).

Xanthippe [mercenaire de Laconie, au service de Carthage, III ${ }^{e}$ s. ; RE, 2] : Xantipus (III.99.4).

Xénocrate de Chalcédoine [philosophe, disciple de Platon, IV $V^{e}$ s. ; RE, 4 / NRE, 2] : Zenocrates (I.109.10).

Xénophon [disciple de Socrate et chef de guerre athénien, $\left.V^{e}-I V^{e} s.\right]$ : Zenefon (I.118.1, II.87.2).

Xerxes [roi de Perse, $V^{e}$ s.] : Xerces (III.48.2).

Zénon de Citium [philosophe stoïcien, $I V^{e}-I I I^{e} s$. ; RE/NRE, 2] : Zenon (III.35.6). 